\title{
The Black Hole Mass vs Bulge Mass Relationship in Spiral Galaxies
}

\author{
Alessandro Marconi ${ }^{1}$, David Axon ${ }^{2}$, John Atkinson ${ }^{2}$, James Binney ${ }^{3}$, \\ Alessandro Capetti ${ }^{4}$, Marcella Carollo ${ }^{5}$, Linda Dressel ${ }^{6}$, Holland \\ Ford $^{7}$, Joris Gerssen ${ }^{6}$, Mark Hughes ${ }^{2}$, Duccio Macchetto ${ }^{6}$, Witold \\ Maciejewski $^{3}$, Michael Merrifield ${ }^{8}$, Claudia Scarlata ${ }^{6}$, William \\ Sparks $^{6}$, Massimo Stiavelli ${ }^{6}$, Zlatan Tsvetanov ${ }^{7}$, Roeland van der \\ Marel $^{6}$ \\ ${ }^{1}$ Osservatorio Astrofisico di Arcetri, L.go Fermi 5, I-50125 Firenze, Italy \\ ${ }^{2}$ Department of Physical Sciences, University of Hertfordshire, UK \\ ${ }^{3}$ Theoretical Physics, University of Oxford, UK \\ ${ }^{4}$ Osservatorio Astronomico di Torino, Italy \\ ${ }^{5}$ Columbia University, Department of Astronomy, USA \\ ${ }^{6}$ Space Telescope Science Institute, USA \\ ${ }^{7}$ Center for Astrophysical Sciences, Johns Hopkins University, USA \\ ${ }^{8}$ School of Physics $\&$ Astronomy, University of Nottingham, UK
}

\begin{abstract}
We describe an on-going HST program aimed at determining the relationship between the nuclear black hole mass and bulge mass in spiral galaxies. We have selected a volume limited sample of 54 nearby spiral galaxies for which we already have ground based emission line rotation curves, CCD surface photometry and radio maps. We are now obtaining HST/STIS longslit observations of each of the galaxies in the sample in order to determine the nuclear $\mathrm{H} \alpha$ rotation curve at high $\left(\sim 0^{\prime \prime} 1\right)$ spatial resolution. We will use these data to measure the unresolved dark mass concentration at the nucleus of each object. Here we show the first results from observations of objects in the sample.
\end{abstract}

\section{Introduction}

It has long been suspected that the most luminous AGN are powered by accretion of matter onto massive black holes (MBH). This belief, combined with the observed evolution of the space-density of AGN (van der Marel 1999 and references therein) and the high incidence of low luminosity nuclear activity in nearby galaxies (Ho, Filippenko, \& Sargent 1997), implies that a significant fraction of luminous galaxies must host black holes of mass $10^{6}-10^{10} \mathrm{M}_{\odot}$.

It is now clear that a large fraction of hot spheroids contain a $\mathrm{MBH}$ (e.g. Harms et al. 1994; Macchetto et al. 1997; van der Marel et al. 1998; Richstone et al. 1998). Moreover, there is a suggestion in the data that the hole mass is proportional to the mass (or luminosity) of the host spheroid. Quantitatively, $M_{\mathrm{BH}} / M_{\mathrm{sph}} \approx 0.5 \%$ (e.g. Richstone et al. 1998). This relation is still controversial, however, both because the sensitivity of published searches is correlated 
with bulge luminosity, and because there is substantial scatter in $M_{\mathrm{BH}}$ at fixed $M_{\text {sph }}$. Recently Ferrarese \& Merrit (2000) and Gebhardt et al. (2000) have shown that a tighter correlation holds between the $\mathrm{BH}$ mass and the velocity dispersion of the bulge. Clearly, any correlation of black hole and spheroid properties would have important implications for theories of galaxy formation in general, and bulge formation in particular.

To date the majority of $\mathrm{BH}$ detections have taken place in luminous, earlytype systems, and most of the data for spirals come either from studies of water masers, or are for nearby spirals in which a MBH can be detected through stellar dynamics. A more systematic study of spiral galaxies is clearly needed at this stage.

\section{Description of the sample}

Prompted by the above motivations, we are undertaking a comprehensive survey for $\mathrm{MBHs}$ in spiral galaxies, both quiescent and active. We identified a volume limited sample $(V<2000 \mathrm{~km} / \mathrm{s})$ of $54 \mathrm{Sb}, \mathrm{SBb}, \mathrm{Sc}$, and $\mathrm{SBc}$ spiral galaxies from a ground-based study by Axon et al. who obtained $\mathrm{H} \alpha$ and [NII] rotation curves at seeing-limited resolution of $1^{\prime \prime}$ for 128 spiral galaxies from the RC3 catalogue. The selected spiral galaxies are known to have nuclear gas disks and span wide ranges in bulge mass and concentration. The low redshift cut-off was chosen to ensure a high spatial resolution on source in order to detect even lowmass MBHs. The frequency of AGN in our sample is typical of that found in other surveys of nearby spirals, with comparable numbers of weak nuclear radio sources and LINERS.

\section{Aims of the project}

The main aim of our survey is to verify if the correlations of the $\mathrm{BH}$ mass with bulge mass and velocity dispersion (Richstone et al. 1998, Ferrarese \& Merrit 2000, Gebhardt et al. 2000) also hold for spiral galaxies. Recently, it has been shown that hierarchical galaxy formation models can account for these correlations (Haehnelt \& Kauffmann 2000). If massive spheroids form by hierarchical merging, then the growth of MBHs may be also a hierarchical process. Small spheroids have undergone less mergers and are thus expected to have smaller BHs. Thus, the above correlations may hold the key to testing these ideas about galaxy evolution. Furthermore, one would like to compare the $\mathrm{BH}$ mass with other galaxy properties such as the shape of the nuclear light profiles; a comparison with AGN activity hosted in the galaxy would provide constraints on the efficiency of accretion.

\section{Observations}

For each galaxy, we obtain three medium resolution spectra with STIS and the G750M grating centered on $\mathrm{H} \alpha$ using the $0{ }^{\prime} 1$ or $0^{\prime \prime} 2$ slit. The resolution is thus $\mathcal{R} \sim 6000$ or 3000 respectively. The spectra are taken for three parallel slit positions, with the central one centered on the galaxy nucleus and the other two 

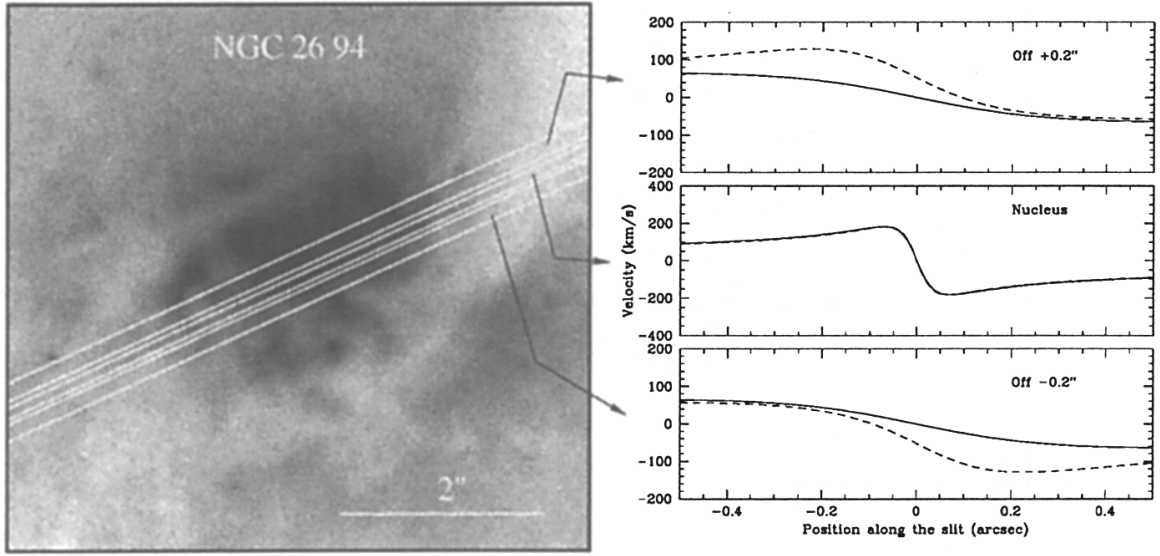

Figure 1. Three parallel slit positions with the central one on the galaxy nucleus constrain the line of nodes of the nuclear gas disk. The solid and dashed lines in the right panel correspond to a combination of different $\mathrm{BH}$ masses and angle between the slit and the line of nodes of the disk which yields the same rotation curve on nucleus. This degeneracy can be resolved by looking at the off-nuclear positions.

offset by $\pm 0 ! 2$. The reason for this choice is explained in Fig. 1 . In order to remove cosmic rays and hot pixels, we obtain two exposures for each slit position with the object shifted by an integer number of detector pixels along the slit. The use of HST guarantees a spatial resolution of about 0 "' 1 which corresponds to $10 \mathrm{pc}$ on a galaxy at a distance of $20 \mathrm{Mpc}$.

\section{Preliminary results}

We currently have HST/STIS data for $90 \%$ of the sample and have extracted rotation curves for these galaxies. Generally, the rotation curves show a steep nuclear rise to a long plateau. In several cases the nuclear rotation curve presents the characteristic unresolved S-shaped velocity structure expected for Keplerian rotation around a mass concentration (Fig. 2). This dynamical signature is accompanied by an abrupt increase of the emission line brightness toward the center, indicative of the presence of a well defined morphological structure, very likely a rotating circumnuclear disk. Comparison with the stellar brightness profiles derived from the STIS acquisition images yields stringent lower limits on the $\mathrm{M} / \mathrm{L}$ ratios that strongly suggest the presence of a supermassive black hole. To interpret the observed rotation curves we have software in place to calculate and fit to the data the predictions of thin disk models with circular rotation (e.g., Marconi et al. 2000; van der Marel \& van den Bosch 1998).

As an example, we now focus on NGC 4041, which clearly shows the possibilities of this project. NGC 4041 is at a distance of about $20 \mathrm{Mpc}$. The rotation curve shown in the right panel of Fig. 2 shows the S-shaped signature of a ro- 

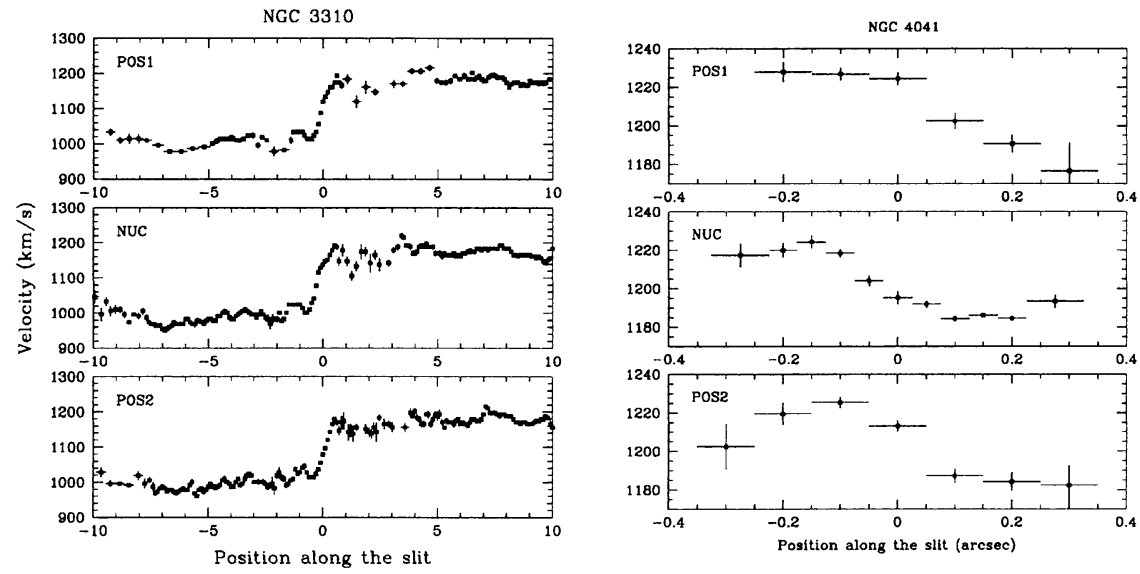

Figure 2. Rotation curves of NGC 3310 (left) and NGC4041 (right). Note the small amplitude of the NGC 4041 curves $(\sim 40 \mathrm{~km} / \mathrm{s})$.

tating disk of $\sim 30 \mathrm{pc}$ radius. Note the small velocity amplitude of the rotation curve which is about $40 \mathrm{~km} / \mathrm{s}$ peak to peak. Assuming an edge-on disk with the line of nodes parallel to the slit one can infer that the minimum dynamical mass required to explain the data is $\sim 3 \times 10^{6} \mathrm{M}_{\odot}$. This mass estimate takes into account the "smearing" of the rotation curve due to the finite slit size and instrumental point spread function. This low mass value gives an indication of the lowest masses which can be detected with our project. The inferred dynamical mass for NGC 4041 should be considered an upper limit to the mass of a possible $\mathrm{MBH}$, since the nuclear star cluster in this galaxy may be responsible for all or part of the dynamically inferred mass.

\section{References}

Ferrarese, L., Merrit, D. 2000, ApJ, 539, L9

Gebhardt, K., et al. 2000, ApJ, 539, L13

Haehnelt, M. G., Kauffmann, G. 2000, MNRAS, 318, L35

Harms, R. J., et al. 1994, ApJ, 435, L35

Ho, L.C., Filippenko, A.,V., Sargent, W.L.W. 1997, ApJ, 487, 568

Macchetto, D., Marconi, A., Axon, D.J., Capetti, A., Sparks, W.B., Crane, P. 1997, ApJ, 489, 579

Marconi, A., Capetti, A., Axon, D., Koekemoer, A., Macchetto, D., Schreier, E. 2000, ApJ, in press (astro-ph/0011059)

Richstone, D., Ajhar, E.A., Bender, R., et al. 1998, Nature, 395, A14

van der Marel, R.P. 1999, in Proceedings of IAU Symp. 186, Sanders D.B., Barnes J., eds., Kluwer Academic Pub., p. 333-340

van der Marel, R. P., Cretton, N., de Zeeuw, P., Rix, H.-W. 1998, ApJ, 493, 613

van der Marel, R. P., van den Bosch, F. C. 1998, AJ, 116, 2220 